\title{
A Standard-Based Software Infrastructure to Support Power System Protection in Distributed Energy Systems
}

\author{
José Lima $^{1}$, Vasco Gomes ${ }^{1}$, João Martins ${ }^{1}$, and Celson Lima ${ }^{1,2}$ \\ ${ }^{1}$ CTS, Uninova, Dep. ${ }^{\circ}$ Eng. ${ }^{a}$ Electrotécnica, Faculdade de Ciências e Tecnologia, FCT, \\ Uiversidade Nova de Lisboa, Portugal \\ \{jose.a.o.lima, vascomdgomes\} @gmail.com, \\ jf.martins@fct.unl.pt \\ ${ }^{2}$ Instituto de Ciências e Geociências, Universidade Federal do Oeste do Pará, Brazil \\ cpl@uninova.pt, celsonlima@ufpa.br
}

\begin{abstract}
Protection in distributed energy systems depends on the operation of a set of coordinated protective devices spread around the power system. Usually these devices encompass some communication skills and can be remotely operated by energy management systems. Despite this interaction readiness, most of them rely only on non-standard communication protocols and some interoperability issues still remain. This paper presents an innovative plug \& play approach for the integration of protective devices, supported by a standard-based and service-oriented software infrastructure entitled NEMO. This approach adopts the IEC 61850 semantic information model and the DPWS specific communication service mapping, to perform a seamless device integration and communication of protective devices.
\end{abstract}

Keywords: DPWS, IEC 61850, Power Distribution, Power System Protection, Power system management, Networked control systems.

\section{Introduction}

Power grids, where energy is generated, distributed and consumed in a smart and efficient way, are revealing themselves as the energy systems of the future [1]. However, current systems and platforms rely mostly on private or access restricted communication protocols and do not target legacy or multiple vendor installations, or even ad-hoc systems [2].

In order to be able to perform the correct diagnosis and necessary actuation, the energy system must be able to properly take acquaintance of the status of each of its players, and perform the adequate operations.

Nowadays, due to the increase in energy demand, most power systems are being operated very closely to their stability margins. Therefore, protective devices play a central part at addressing stability related problems.

Fault conditions have usually complex origins and behavior, and incorrect protection operation or even delayed fault clearance may be devastating to the power system. Therefore it is imperative that protective devices are correctly monitored and 
operated to guarantee grid stability. Moreover, they can contribute with relevant information for the operational decision process.

The NEMO system, further described in [3], aims to enable the seamless integration and communication of every device (related with distributed energy systems) plugged into the grid, despite its manufacturer or communication capabilities, using communication standards such as IEC 61850 and Devices Profile for Web Services (DPWS) [4].

This paper focuses on the development of a plug-and-play environment for the integration and operation of protective devices, using NEMO architecture. The next sections describe the several stages regarding device specification and communication, and the respective implementation, using the IEC 61850 data model allied to the DPWS service mapping, for the integration and operation of protective devices.

\section{Contribution to Value Creation}

Energy industry has recently witnessed a growing scientific interest and research on distributed generation [5-7]. The worldwide call for increasing energy efficiency, due to the obvious global climate change and to the continuous quest for lower electrical energy bills, has influenced the introduction of a holistic perspective into electrical system analysis. Developing new concepts and tools for intelligent energy management, has become one of today's major challenges.

This work intends to perform its contribution for value creation by exploring the next generation of network-enabled devices for the purpose of dynamically and remotely monitor and control aggregations of protective IEDs, thus improving energy management performance.

\section{The NEMO Conceptual Architecture}

NEMO intends to create an architecture that becomes a facilitator regarding the management of complex systems where production, distribution and consumption of energy from renewable sources are considered [8].

The conceptual vision guiding NEMO is that networks of energy-related devices can be operated with the help of a (distributed) software infrastructure based on service oriented paradigm and standards.

Any instance of NEMO can use both new and legacy IEDs, which are required to have a minimum level of 'intelligence' in order to be virtualised. In other words, they have to provide a software-enabled communication channel to be used in a communication process.

Two networks, namely energy and software networks compose each so-called NEMO system. The former is formed by systems \& devices producing, distributing, and consuming energy. The later used to monitor and control the energy network.

Two basic issues are addressed: recognition of IEDs and communication with them. NEMO strategy to overcome them relies on two main pillars, namely SOA and 
Standards. On the one hand, SOA architectural guidelines are followed for handling all IEDs as 'service providers' and rely on DPWS profile for communication with and among those IEDs. On the other hand, IEDs are modeled, configured, and characterized into the System using the IEC 61850 standard. The role of both DPWS and IED 61850 are described in detail in the next section.

The NEMO software network, shown in Fig. 1, relies on five main components, namely: Kernel, API, Bus, Connector, and IEDs.

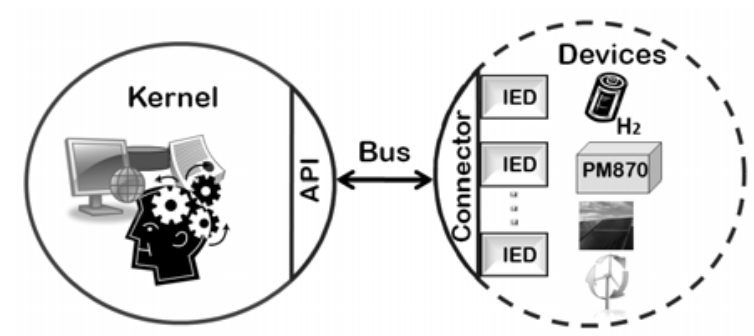

Fig. 1. The main components of NEMO Software Network

IEDs are at the nearest level regarding the devices and through them, commands are sent to the devices or data is gathered from them.

On the opposite side, there is the Kernel (NEMO-K), which is the supervisor of the operation of Software Network. The NEMO-K manages the services that can be provided by the system to the rest of the world. These services are named External Services, implemented as web services.

The Bus (NEMO-B) supports the interaction between the NEMO-K and all devices that are connected in the software network. Finally, the Connector (NEMO-C) is a wrapper that allows both integration and virtualisation of IEDs into a given NEMO system.

Each of these components is further explained in [9], and together they are essential for supporting the described conceptual approach.

\subsection{Standard-Based Approach}

IEC 61850 is a worldwide-accepted standard for handling communication within substations. It integrates an information model, the so-called Abstract Communication Service Interface (ACSI), for substation description and the Substation Configuration Language (SCL), used to describe the ACSI information model.

ACSI allows describing an energy system and its respective components in a standard manner, independently from the respective individual manufacturers and with high level of detail. NEMO takes advantage of the intrinsic ACSI ability of virtualizing IEDs, by decomposing their respective physical properties and functionalities into a data model [9]. IED vistualisation using the ACSI data model is further detailed in [3]. 
Each standard compliant IED carries an XML-based SCL file, where the entire respective ACSI information is stored.

The physical features of each device may be enabled and disabled, or its information may be requested or changed, through the invocation ACSI services.

A shown in Fig. 2, two main ACSI service types are considered by NEMO: GetDataValues and SetDataValues. While the former is invoked for monitoring operations, when knowledge about the state of a physical feature is required, the later allows the physical control of a given device, replacing the older data attribute value by a new one.

Both require a reference that points to the required Data Attribute path with which the service is to be invoked. This reference is given by a Functional Constraint Data Attribute (FCDA) which includes, among others, the Logical Device (LD), Logical Node (LN), Data Object (DO), and Data Attribute (DA) [3] that univocally characterise the physical operation (monitoring or control) to be performed.

DPWS, the Web Service standard promoted by OASIS, was chosen to support the operation of the channel and really allow a seamlessly communication among all members of the network, supporting the inter-devices communication [10][11]. Web Services are the preferred mechanism for SOA implementation [12][13] and the application of Web Services at device level will improve the operation of the system as well as the development process [14]. The service mapping between ACSI and DPWS allows supporting higher level heterogeneous platforms.

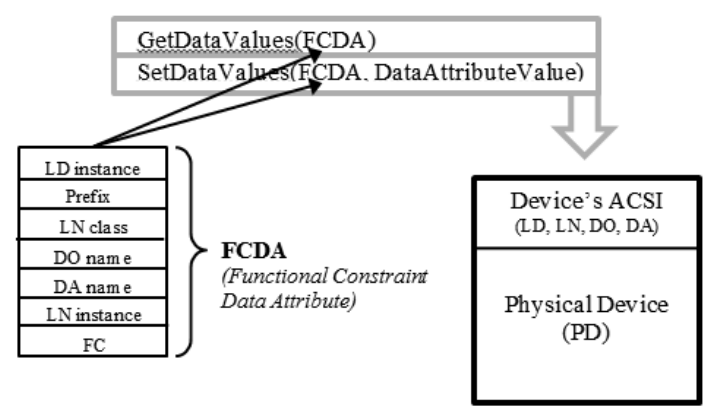

Fig. 2. ACSI services invocation

Similar to the Specific Communication Service Mapping (SCSM) based on MMS, described in IEC 61850[15], NEMO uses a SCSM based on DPWS. The aforementioned ACSI Services - GetDataValues(FCDA) and SetDataValues(FCDA, DataAttributeValue) - are identified and mapped into the GetIEC(FCDA) and PutIEC(FCDA, newValue) web services (NEMO Internal Services), respectively. Therefore, each web service will be able to interact with single or multiple low level device physical features, through the invocation of NEMO Internal Services, each of them identified by its ACSI path.

Additionally to the services specified by the IEC 61850 data model, and in order to provide advanced features to the substation automation system not considered by the standard, NEMO defines a NEMO Communication Service Interface (NCSI)[3]. 
NCSI incorporates two NEMO Internal Services: GetNonIEC(NemoIS): and PutNonIEC(NemoIS, newValue). These additional NEMO Services allow the integration and request of non-compliant services.

Since the majority of the IEDs do not understand DPWS, they usually need a mediator to make a bridge between DPWS and ACSI. This translation process is also performed by the NEMO Connector who is responsible for offering device's features in the form of Web Services, performing all the necessary mapping between the device's ACSI and DPWS. This process is further described in [9].

\section{The Experimental Setup}

An instance of NEMO System was implemented and evaluated in the experimental scenario [9]depicted in Fig. 3, which covers the whole energy process. For the sake of clarity, only the distribution part is explored and detailed here, which includes a digital protective relay (SEPAM).

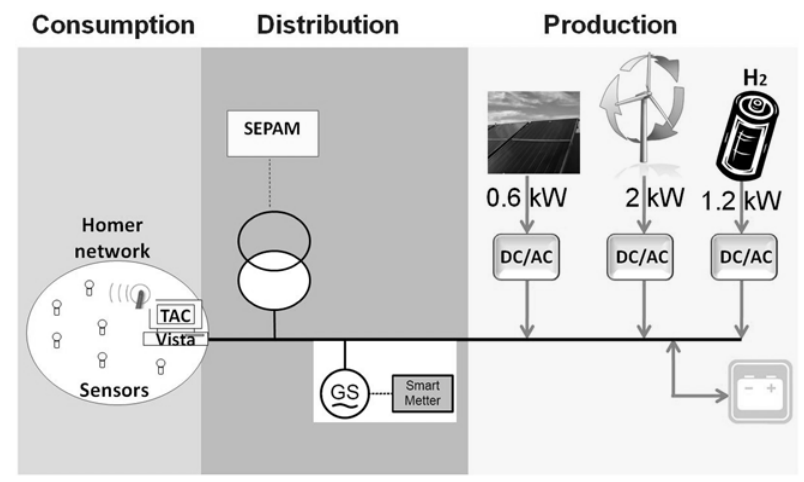

Fig. 3. The NEMO experimental setup [9]

To stand out as a real and challenging test, the chosen digital protective relay doesn't comply with the DPWS and IEC 61850 standards. Interaction among all noncompliant devices integrated into the NEMO Software Network requires the use of proprietary communication protocols. For the SEPAM case, since the proprietary protocol is Modbus two communication mappings have to be performed, namely:

- $\quad$ Modbus to IEC 61850, for device description;

- IEC 61850 to DPWS, introducing web service invocation capabilities into the device.

In order to perform this mapping, a NEMO-C is attached to each non-compliant device, guaranteeing IED compliancy.

For addressing the assessment of the SEPAM integration, the following tests were performed: Reading values from the SEPAM, resetting the SEPAM after a peak of current and resetting of the maximum values for the measured electrical current, read 
the errors available in SEPAM, opening and closing the SEPAM's circuit breaker, retrieving information about tripping occurrences, retrieve thermal protections alarms status, measuring the earth fault current, and measuring current peaks and instant values.

\section{Current Implementation}

As previously described, the scope of this work is the to develop a plug\&play approach for remotely monitoring and operating electrical protective devices and, therefore, only the distribution part of the experimental setup will be detailed, as shown in Fig. 4. The production and consumption parts are further detailed in [3],[9].

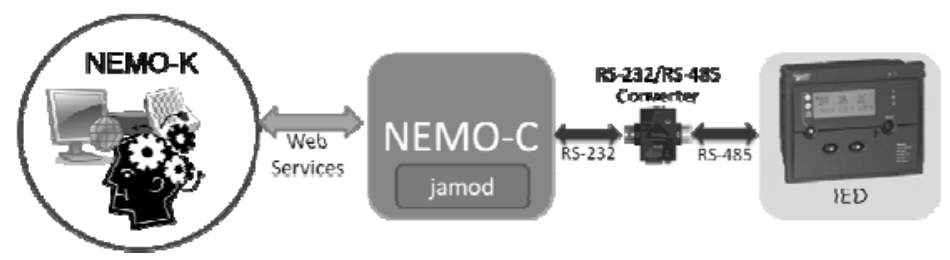

Fig. 4. Current distribution implementation scenario

As aforementioned, NEMO-C is responsible for the integration and virtualization of IEDs into the NEMO System, providing additional features through Web Services and enabling the interaction between NEMO system and the physical devices.

Three different types of mappings are performed by the NEMO-C:

- Between DPWS and IEC 61850, to allow the invocation of ACSI services using DPWS web services to both IEC 61850 compliant and non-compliant devices;

- Between IEC 61850 and the IED's manufacturer communication protocol, to guarantee that IEC 61850 non-compliant devices understand ACSI services;

- Between DPWS and the IED's manufacturers communication protocol, to allow the invocation of services not contemplated by the IEC 61850 .

NEMO-C receives the DPWS service invocations of the NEMO-K and through internal the mappings, it will be capable of performing the requested operations in the device.

Regarding the distribution energy area, and for the purpose of this work, NEMO-C is connected to a SEPAM digital relay. The connection with the SEPAM is performed through a RS-232-RS-485 connector. The Jamod JDK library, which is used for implementing the Modbus protocol, assists the data exchange between the master (NEMO-C) and the slave (SEPAM), which communicates via RS485.

Examples of ACSI compliant services and of non-ACSI compliant services currently implemented in the SEPAM are shown in Tables 1 and 2, respectively. 
Table 1. Examples of ACSI services currently implemented in SEPAM

\begin{tabular}{|c|c|c|c|c|c|c|}
\hline \multicolumn{6}{|c|}{ FCDA } & \multirow{2}{*}{ NEMO Internal Service } \\
\hline LdInst & LnClass & LnInst & DoName & DaName & $\mathrm{Fc}$ & \\
\hline SEPAM & MMXN & 1 & Tmp & mag & MX & Phase Current IB \\
\hline SEPAM & PTTR & 1 & Tmp & mag & MX & Thermal Capacity Used \\
\hline SEPAM & XCBR & 1 & Pos & stVal & ST & Open Circuit Breaker \\
\hline SEPAM & XCBR & 2 & Pos & stVal & ST & Close Circuit Breaker \\
\hline SEPAM & PTTR & 1 & AlmThm & stVal & ST & $\begin{array}{c}\text { Protection } 49 \text { RMS } \\
\text { thermal alarm }\end{array}$ \\
\hline SEPAM & SCBR & 1 & SwA & mag & MX & Phase Tripping Current \\
\hline
\end{tabular}

Table 2. Non-ACSI services currently implemented in SEPAM

\begin{tabular}{||l|l|l|l||}
\hline \hline Type of Service & \multicolumn{1}{|c|}{ NEMO Service Identifier } & Input Units & Output Units \\
\hline \hline Get & Serial Number & n/A & n/A \\
GetPut & Reset & n/A & n/A \\
GetPut & ResetPhasePeakDemandCurrentValues & n/A & n/A \\
\hline
\end{tabular}

Lets consider that the NEMO-K requests an invocation of a non-ACSI service, for instance, to reset the SEPAM after a peak of current. This operation starts with the invocation of the PutNonIEC web service having NEMO service identifier parameter with the value "Reset" and the new_value parameter with the data logic value one (Table 2). This web service request will be received in the NEMO.IS component, and since it is identified as non-compliant service, it is forwarded to NEMO.IS.to.NonACSI, where its accomplishment is verified. If NEMO-C is able to execute the requested service, this is dispatched to the NonACSI.to.IED component, which will perform the requested operation through the Communicator component, according to the NEMO-C internal mapping. Otherwise the service will be denied.

Lets consider now anIEC 61850 compliant monitoring operation, regarding the thermal capacity used by the SEPAM. AGetIEC service is invoked (ACSI compliant), introducing the reference PTTR.Tmp.mag[MX] to the FCDA parameter (Table 1). NEMO-K invokes the NEMO.IS, and this time the request is dispatched to the NEMO.IS.to.ACSI component, which verifies if the FCDA is correct, i.e., if it is defined in the IED's SCL file. If this is verified and the IED is IEC 61850 compliant, the operation is performed according to the IEC61850 protocol. Otherwise, if the IED is not IEC 61850 compliant, the ACSI.wrapper component acts and the service is performed according to the FCDA internal mapping.

\section{Conclusion and Open Points}

Power system protection is a critical area concerning power systems operation. A holistic view regarding the continuous and remotely management of protective devices is, therefore, a must. With the NEMO architecture presented in this paper, this 
goal can be achieved. By integrating two communication standards and a standard data model, NEMO provides the seamless integration and interoperability of protective devices, required for a real effective operational architecture for power system protection.

As future work, it is planned the implementation of additional services essential to the distribution area, as well as the implementation of a mapping between the Eventing DPWS [11] feature and the Report ACSI service [15].

\section{References}

1. Vale, Z.A., Morais, H., Khodr, H.: Intelligent Multi-Player Smart Grid Management Considering Distributed Energy Resources and Demand Response. Power, pp. 1-7 (2010)

2. NIST Special Publication 1108, Framework and Roadmap for Smart Grid Interoperability, Release 1.0, National Institutes of Standards and Technology (2010)

3. Lima, J., Lima, C., Gomes, V., Martins, J.F., Barata, J., Ribeiro, L., Cândido, G.: DPWS as Specific Communication Service Mapping for IEC 61850. In: INDIN 2011 - IEEE 9th International Conference on Industrial Informatics, Lisbon (2011)

4. Devices Profile for Web Services Version 1.1 Specification, http://docs.oasisopen. org/ws-dd/dpws/1.1/os/wsdd-dpws-1.1-spec-os . html

5. Jin, T., Jimenez, J.J.: A Review on Planning and Automation Technologies for Distributed Generation Systems. Power, 269-274 (2010)

6. Bai, J., Xiao, H., Yang, X., Zhang, G.: Study on Integration Technologies of Building Automation Systems based on Web Services. Electrical Engineering (2009)

7. Carr, J.A., Balda, J.C., Mantooth, H.A.: A Survey of Systems to Integrate Distributed Energy Resources and Energy Storage on the Utility Grid. Energy (2008)

8. Lima, C., Martins, J.F., Barata, J., Ribeiro, L., Cândido, G.: Towards a service based infrastructure to improve efficiency into energy systems: The NEMO\&CODED quest. In: 10th IFAC Workshop on Intelligent Manufacturing Systems, Lisbon (2010)

9. Lima, C., Gomes, V., Lima, J., Martins, J.F., Barata, J., Ribeiro, L.: Cândido. G.: A Standard-based Software Infrastructure to Support Energy Efficiency Using Renewable Energy Sources. In: 20th IEEE International Symposium on Industrial Electronics, Gdansk, pp. 1175-1180 (2011)

10. Cândido, G., Jammes, F., Barata, J., Colombo, A.: Generic Management Services for DPWS-enabled devices. In: IECON 2009 - Annual Conference of the IEEE Industrial Electronics Society (2009)

11. Devices Profile for Web Services Version 1.1 Specification, http://docs.oasisopen.org/ws-dd/dpws/1.1/os/wsdd-dpws-1.1-spec-os.html

12. Ribeiro, L., Barata, J., Mendes, P., Azevedo, A.: MAS and SOA: Complementary Automation Paradigms. In: Azevedo, A. (ed.) Innovation in Manufacturing Networks. Springer, Heidelberg (2008)

13. Cândido, G., Barata, J., Colombo, A., Jammes, F.: SOA in reconfigurable supply chains: A research roadmap. In: Engineering Applications of Artificial Intelligence (2009)

14. Jammes, F., Smit, H.: Service-oriented paradigms in industrial automation. IEEE Transactions on Industrial Informatics 1(1), 62-70 (2005)

15. IEC 61850 - Communication Networks and Systems in Substations

16. Java Modbus Library (jamod), http: / / jamod. sourceforge.net 